\title{
Technical Advances in Radiation Therapy for Brain Tumors
}

\author{
CLAUDIA SCARINGI ${ }^{1,2}$, LINDA AGOLLI $^{3}$ and GIUSEPPE MINNITI ${ }^{2,4}$ \\ ${ }^{1}$ Radiation Oncology Unit, Sant' Andrea Hospital, University "Sapienza", Rome, Italy; \\ ${ }^{2}$ Radiation Oncology Unit, UPMC Hillman Cancer Center, San Pietro Hospital, Rome, Italy; \\ ${ }^{3}$ Department of Radiation Oncology, Faculty of Medicine and Carl Gustav Carus University Hospital, \\ Technical University, Dresden, Germany; \\ ${ }^{4}$ IRCCS Neuromed, Pozzilli, Italy
}

\begin{abstract}
Radiation therapy plays a critical role in the management of brain tumors. Recent advances in radiation techniques include the use of intensity-modulated radiotherapy (IMRT), volumetric-modulated arc therapy (VMAT) and stereotactic radiosurgery (SRS). All of these techniques allow the delivery of higher radiation doses to the target volume, at the same time reducing the risk of toxicity to normal tissues as compared with conventional $3 D$ conformal radiotherapy $(3 D-C R T)$. Proton therapy may represent a treatment alternative to photon irradiation, due to the more favorable dose distribution to the target volume. This review summarizes current developments in radiation therapy and their clinical impact on the management of patients with brain tumors.
\end{abstract}

Radiation therapy plays a critical role in the management of primary and secondary brain tumors. In the past few decades, radiotherapy (RT) has seen technical advances in all aspects of treatment, with improvement in patient immobilization, imaging, treatment planning and delivery. Advances in imaging and RT technology have enabled more precise tumor localization and dose delivery, leading to a reduction in the volume of normal brain tissue irradiated at high radiation doses. The principal aim of normal tissue sparing is to reduce the potential long-term toxicity of RT while maintaining its effectiveness. Radiation techniques have evolved from 3D

This article is freely accessible online.

Correspondence to: Claudia Scaringi, MD, Radiation Oncology Unit, UPMC Hillman Cancer Center, San Pietro Hospital, Via Cassia 600, 00189 Rome, Italy. Tel: +39 0688846800, e-mail: scaringic@upmc.edu

Key Words: Brain tumors, conformal radiotherapy, IMRT, VMAT, stereotactic radiosurgery, IGRT, proton therapy, review. conformal radiotherapy (3D-CRT) to intensity-modulated radiotherapy (IMRT), volumetric-modulated arc therapy (VMAT), and stereotactic techniques, including either stereotactic radiosurgery (SRS) or fractionated stereotactic radiotherapy (FSRT). A further improvement in accuracy of radiation delivery has been made with the development of image-guided radiation therapy (IGRT), in which image guidance enables better precision in patient setup and target localization, allowing reduction of planning margins and leading to a smaller volume of healthy tissue irradiated at high doses. Currently, there is interest in the use of particle therapy for treating brain tumors because of the ability to concentrate the dose of protons and ions in the target volume while simultaneously sparing surrounding healthy tissues.

We provide a short review to summarize recent technical advances for irradiation of brain tumors, as well as highlight the potential benefits in terms of tumor control and reduction of long-term toxicity of different radiotherapy techniques.

\section{IMRT and VMAT}

Intensity-modulated radiation therapy represents the main evolution of 3D-CRT, in that it allows a superior dose distribution to the target volume and the delivery of highly conformal irradiation to concave and irregularly shaped volumes (1). Unlike conventional RT, the intensity of the radiation beams is delivered as a sequence of many small beams modulated by a multi-leaf collimator (MLC) in a dynamic sweeping manner (sliding window) or in a step-andshoot manner, by using sophisticated computerized optimization planning (inverse planning). In clinical practice, the radiation oncologist delineates the target volume and specifies the prescribed radiation dose to the target and organs at risk (OARs). Based on these parameters, computer software optimizes the motion trajectories or segment shapes of the MLC to achieve intensity modulation, with the aim of 
improving dose conformity to the target while minimizing radiation exposure to surrounding normal tissue and OARs. In addition, the IMRT technique can be used to deliver different doses of radiation to different target volumes in a single phase as simultaneous integrated boost. Tomotherapy is a rotational form of IMRT in which dose conformity to the tumor is achieved by modulating the intensity of a rotating fan beam using MLCs (2). During treatment delivery, the patient is moved through the bore of the machine, while the gantry simultaneously rotates around him. A further evolution of IMRT technique is represented by VMAT, in which the radiation dose is continuously delivered as the gantry of the linear accelerator (LINAC) rotates around the patient through single or multiple arcs (3). VMAT provides a highly conformal dose distribution to the tumor, by modulating the intensity of the radiation beam, the dose rate and the gantry rotation speed, while shortening the treatment time and reducing the number of monitor units delivered, compared to conventional IMRT.

A recent advance in photon irradiation is represented by a new generation of LINAC with flattening filter (FF)-free beams (4). The removal of the FF allows an increase in dose rate up to four times compared to traditional FF beams. Therefore, the consequently shorter time of delivery can potentially reduce errors and obtain higher treatment accuracy, at the same time improving patient comfort.

The increased conformality achieved with IMRT and VMAT techniques may allow escalated tumor doses or hypofractionated radiation schedules without increasing toxicity to normal tissue, and thus may improve local tumor control and survival.

IMRT and VMAT are increasingly used for hippocampal sparing in patients undergoing brain irradiation for primary and secondary brain tumors, with the aim of avoiding longterm worsening of neurocognitive function (5). In patients with medulloblastoma, treatment with IMRT can achieve a lower rate of hearing loss and neurocognitive decline as compared with 3D-CRT (6-8). When used for either low-grade or high-grade gliomas, IMRT and VMAT allow for improved target conformity and better critical tissue sparing, including the hippocampus and brainstem; this might result in reduced late toxicities associated with RT. While the dosimetric superiority of IMRT and VMAT over 3DCRT for brain tumors has been clearly demonstrated for different tumor types (9-10), randomized studies are needed to assess the clinical advantages of these techniques in terms of efficacy and reduction of radiation-induced late toxicities. Concerns have been raised regarding the risk of secondary malignancies, due to higher integral dose to the surrounding healthy tissue with these modern techniques, especially for patients with long life expectancy (11). The estimated incidence of the risk for secondary cancer using IMRT for brain tumor treatment is approximately 130/10,000 persons/year (12). Longer follow- up and larger series are needed to report the real risk of late effects and clinical outcomes for IMRT and VMAT techniques.

\section{Stereotactic RT}

Stereotactic irradiation represents a further refinement of CRT, with the advantage of improved patient immobilization achieved with the use of either a frame-based or a frameless mask stereotactic system, leading to submillimetric accuracy of patient repositioning. The radiation dose can be delivered as single-fraction, multi-fraction SRS (2-5 fractions), or as FSRT when conventional fractionation of 1.8-2.0 Gy per fraction is used. The main advantage of SRS is the ability to deliver a highly localized irradiation, as compared to conventional RT, leading to a reduction of the volume of normal brain tissue irradiated at high radiation doses, and minimizing the risk of the long-term consequences of treatment (13).

SRS can be delivered with the use of either a cobalt-60 gamma radiation-emitting source (Gamma Knife ${ }^{\text {TM }}$; Elekta Inc., Stockholm, Sweden) or a LINAC. In its most used form, the Gamma Knife contains 201 small cobalt-60 sources of gamma rays arrayed in a hemisphere within a thickly shielded structure. A primary collimator aims the radiation emitted by these sources to a common focal point. A second external collimator helmet, which fits within the primary collimator, has an array of removable tungsten collimators (one per source) with circular apertures from 4-18 $\mathrm{mm}$ in size. For large and complex-shaped tumors, multiple radiation spheres can be used to cover the entire target in a multiple isocenter technique. The best tumor conformality is achieved by a different combination of number, size and position of the collimators. The dose is typically prescribed at $50 \%$ to obtain the maximum dose at the center of each pinpointed target and minimal dose at the target edge. In LINAC SRS, the dose is delivered with the use of multiple arcs or beams resulting in a similar high dose differential between the target and normal brain tissue. Isodose gradients can be improved using intensity modulation of the beams, restriction of gantry angles and arc lengths, microcollimation, and multiple-isocenter plans. The use of VMAT for SRS with FF-free LINACs allows reduction of overall treatment time, as compared with delivery using conventional FF beams (14). CyberKnife (Accuray, Sunnyvale, CA, USA) is a relatively new technological device which combines a mobile miniaturized LINAC mounted on a robotic 6-degree-of-freedom treatment arm, with an image-guided robotic system. Patients are fixed in a thermoplastic mask and the system allows for either single-fraction or multi-fraction SRS achieving the same level of targeting precision as for framebased SRS. 
SRS is frequently employed to treat both malignant and benign brain tumors and, regardless of the superiority claimed for each of these techniques in terms of dose homogeneity and conformality, the reported clinical efficacy and toxicity are similar (15). The role of SRS in the management of brain metastases is rapidly evolving, and SRS has been widely adopted as an alternative to whole-brain radiotherapy to treat patients with up to 10 brain metastases, with the aim of reducing the risk of neurocognitive impairment $(16,17)$. LINAC-based SRS is usually performed using a single isocenter to treat each lesion. Recently, the use of a singleisocenter technique for the simultaneous treatment of multiple brain metastases, in a single or few sessions, has been evaluated. Preliminary results are promising, showing an improvement in the efficiency of the delivery, while reducing overall treatment time and maintaining a high local control rate (18). SRS or hypofractionated stereotactic RT alone or in association with chemotherapy have been used as salvage therapy for patients with recurrent or progressive malignant gliomas, with a reported median survival of 6-11 months $(19,20)$. For patients with benign brain tumors, including meningiomas, pituitary adenomas, craniopharyngiomas, and acoustic neuromas, local control after either SRS or FSRT is in the range of $80-95 \%$ at 5-10 years, with a low incidence of long-term toxicity (21-24).

\section{IGRT}

Radiation delivery with IMRT or VMAT allows a steep gradient between the target volume and surrounding tissues obtaining a dosimetric benefit. Accurate image guidance is required during delivery of radiation because there is a risk of geographical miss of tumors or overdose to OARs. IGRT is an advanced imaging technology that improves the safe clinical application of modern RT techniques (25). IGRT generally means the use of frequent imaging of the patient within the RT treatment room, with the aim of improving the precision of radiation delivery throughout a process of image acquisition, matching with the reference images and setup corrections. Daily IGRT-based setup has been shown to significantly reduce residual errors, and, consequently, planning margins. Currently, several online 2D and 3D imaging devices directly integrated into the LINAC are used, including kilovoltage and megavoltage $\mathrm{x}$-ray imaging, kilovoltage cone beam computed tomography (kV-CBCT), and megavoltage single-slice computed tomography on helical tomotherapy. The simplest online patient positioning verification method is represented by the electronic portal imaging devices, whereby $2 \mathrm{D}$ images are captured on a flat panel behind the patient and matched with the digitally reconstructed radiographs of the treatment planning computed tomographic scan. $\mathrm{kV}$-CBCT utilizes a gantry mounted source with a flat panel detector to take a series of X-rays images and to reconstruct a final 3D image. In megavoltage-computed tomography on helical tomotherapy, IGRT is performed by the treatment beam, which rotates around the patient while the couch moves. Since the images are acquired and fused with the reference images, translational and rotational displacements from the expected target position are calculated by software, and then errors are corrected by moving a $6 \mathrm{D}$ robotic couch. The quality of images is superior with $\mathrm{kV}$-CBCT compared to the megavoltage imaging (26). The recent ExacTrac/Novalis 6D advanced system allows for accurate positioning comparable to invasive mask fixation (27). A modest set-up difference for intracranial tumors was reported ( $<1 \mathrm{~mm}$ for translations, $<1^{\circ}$ for rotations) when the ExacTrac $\mathrm{X}$-ray 6D (NovalisTx) was compared to the routinely used CBCT (28).

\section{Proton RT}

Protons are positively charged elementary particles, with different physical and biological properties compared to conventional photon radiation. The principal characteristic of these charged particles is the delivery of little energy until the end of their range, known as the Bragg peak, with the highest energy deposition in the target volume and no exit dose. Thanks to these fundamental physical properties, proton RT provides excellent dose distributions to the tumor, while significantly lowering the dose to the surrounding normal tissues.

Proton therapy is increasingly used in pediatric patients with central nervous system tumors with the aim of reducing the potential long-term side-effects of radiation on the development of normal brain tissue in children. For adult and pediatric patients receiving craniospinal irradiation for medulloblastoma, protons may significantly reduce the dose to the cochlea, heart, lung, and kidney, as compared with photons (29-31). For chordomas and chondrosarcomas of the skull base, the use of protons has led to superior results in comparison to conventional photon irradiation, resulting in better clinical outcomes with relatively few significant complications, considering the high doses delivered $(32,33)$. An excellent tumor control of about $90 \%$ at 5 years with low long-term radiation-induced toxicity has been reported with the use of protons in patients with craniopharyngiomas, pituitary adenomas, and meningiomas; however, the reported control and toxicity rates are comparable to those observed with the use of stereotactic photon irradiation (34-36). Randomized clinical trials are warranted to demonstrate the superiority of proton therapy to photon irradiation for brain tumors. In addition, there is a need for long-term follow-up data regarding the different potential risk of developing second tumors following photon and proton therapy.

\section{Conclusion}

RT represents an essential part of the treatment of brain tumors and the past decades have seen significant technological 
advances in all aspects of radiation delivery. With improved clinical outcomes of cancer treatment, and an increasing number of long-term survivors, minimizing potential RTrelated toxicities has also become a priority. New radiation techniques, including IMRT, VMAT, stereotactic techniques, and IGRT allow for more precise treatment delivery, doseescalation regimens and altered fractionations that can improve tumor control while reducing the volume of healthy tissue irradiated to high doses, and, hence, minimizing the long-term toxicity of radiation treatment. Further studies are needed to compare different RT techniques, in terms of efficacy and longterm toxicity, and investigate the optimal radiation dose/fractionation for each type of brain tumor.

\section{References}

1 Cho B: Intensity-modulated radiation therapy: A review with a physics perspective. Radiat Oncol J 36: 1-10, 2018. Erratum in: Radiat Oncol J 36: 171, 2018.

2 Rong Y and Welsh JS: Dosimetric and clinical review of helical tomotherapy. Expert Rev Anticancer Ther 11: 309-320, 2011.

3 Teoh M, Clark CH, Wood K, Whitaker S and Nisbet A: Volumetric-modulated arc therapy: A review of current literature and clinical use in practice. Br J Radiol 84: 967-996, 2011.

4 Georg D, Knöös T and McClean B: Current status and future perspective of flattening filter-free photon beams. Med Phys 38 : 1280-1293, 2011.

5 Kazda T, Jancalek R, Pospisil P, Sevela O, Prochazka T, Vrzal M, Burkon P, Slavik M, Hynkova L, Slampa P and Laack NN: Why and how to spare the hippocampus during brain radiotherapy: The developing role of hippocampal avoidance in cranial radiotherapy. Radiat Oncol 9: 139, 2014.

6 Polkinghorn WR, Dunkel IJ, Souweidane MM, Khakoo Y, Lyden DC, Gilheeney SW, Becher OJ, Budnick AS and Wolden SL: Disease control and ototoxicity using intensity-modulated radiation therapy tumor-bed boost for medulloblastoma. Int $\mathbf{J}$ Radiat Oncol Biol Phys 81: e15-20, 2011.

7 Blomstrand M, Brodin NP, Munck Af Rosenschöld P, Vogelius IR, Sánchez Merino G, Kiil-Berthlesen A, Blomgren K, Lannering B, Bentzen SM and Björk-Eriksson T: Estimated clinical benefit of protecting neurogenesis in the developing brain during radiation therapy for pediatric medulloblastoma. Neuro Oncol 14: 882-889, 2012.

8 Pichandi A, Ganesh KM, Jerrin A, Balaji K, Sridhar PS and Surega A: Craniospinal irradiation of medulloblastoma using high precision techniques - a dosimetric comparison. Technol Cancer Res Treat 14: 491-496, 2015.

9 Wagner D, Christiansen H, Wolff $\mathrm{H}$ and Vorwerk $\mathrm{H}$ : Radiotherapy of malignant gliomas: comparison of volumetric single arc technique (RapidArc), dynamic intensity-modulated technique and 3D conformal technique. Radiother Oncol 93: 593-596, 2009.

10 Chen YD, Feng J, Fang T, Yang M, Qiu XG and Jiang T: Effect of intensity-modulated radiotherapy versus three-dimensional conformal radiotherapy on clinical outcomes in patients with glioblastoma multiforme. Chin Med J (Engl) 126: 2320-2324, 2013.

11 Chargari C, Goodman KA, Diallo I, Guy JB, Rancoule C, Cosset JM, Deutsch E and Magne N: Risk of second cancers in the era of modern radiation therapy: Does the risk/benefit analysis overcome theoretical models? Cancer Metastasis Rev 35: 277288, 2016.

12 Patil VM, Kapoor R, Chakraborty S, Ghoshal S, Oinam AS and Sharma SC: Dosimetric risk estimates of radiation-induced malignancies after intensity-modulated radiotherapy. J Cancer Res Ther 6: 442-447, 2010.

13 Kondziolka D, Shin SM, Brunswick A, Kim I and Silverman JS: The biology of radiosurgery and its clinical applications for brain tumors. Neuro Oncol 17: 29-44, 2015.

14 Stieler F, Fleckenstein J, Simeonova A, Wenz F and Lohr F: Intensity-modulated radiosurgery of brain metastases with flattening filter-free beams. Radiother Oncol 109: 448-451, 2013.

15 Ding D, Yen CP, Starke RM, Lee CC and Sheehan JP: Unyielding progress: Recent advances in the treatment of central nervous system neoplasms with radiosurgery and radiation therapy. J Neurooncol 119: 513-529, 2014.

16 Aoyama H, Shirato H, Tago M, Nakagawa K, Toyoda T, Hatano $\mathrm{K}$, Kenjyo M, Oya N, Hirota S, Shioura H, Kunieda E, Inomata T, Hayakawa K, Katoh $\mathrm{N}$ and Kobashi G: Stereotactic radiosurgery plus whole-brain radiation therapy vs. stereotactic radiosurgery alone for treatment of brain metastases: a randomized controlled trial. JAMA 295: 2483-2491, 2006.

17 Yamamoto M, Serizawa T, Shuto T, Akabane A, Higuchi Y, Kawagishi J, Yamanaka K, Sato Y, Jokura H, Yomo S, Nagano O, Kenai H, Moriki A, Suzuki S, Kida Y, Iwai Y, Hayashi M, Onishi H, Gondo M, Sato M, Akimitsu T, Kubo K, Kikuchi Y, Shibasaki T, Goto T, Takanashi M, Mori Y, Takakura K, Saeki N, Kunieda E, Aoyama H, Momoshima S and Tsuchiya K: Stereotactic radiosurgery for patients with multiple brain metastases (JLGK0901): A multi-institutional prospective observational study. Lancet Oncol 15: 387-395, 2014.

18 Ruggieri R, Naccarato S, Mazzola R, Ricchetti F, Corradini S, Fiorentino A and Alongi F: Linac-based VMAT radiosurgery for multiple brain lesions: Comparison between a conventional multi-isocenter approach and a new dedicated mono-isocenter technique. Radiat Oncol 13: 38, 2018.

19 Ryu S, Buatti JM, Morris A, Kalkanis SN, Ryken TC and Olson JJ; AANS/CNS Joint Guidelines Committee: The role of radiotherapy in the management of progressive glioblastoma: A systematic review and evidence-based clinical practice guideline. J Neurooncol 118: 489-499, 2014.

20 Nieder C, Andratschke NH and Grosu AL: Re-irradiation for recurrent primary brain tumors. Anticancer Res 36: 4985-4995, 2016.

21 Kondziolka D, Mathieu D, Lunsford LD, Martin JJ, Madhok R, Niranjan A and Flickinger JC: Radiosurgery as definitive management of intracranial meningiomas. Neurosurgery 62: 5360, 2008.

22 Minniti G, Esposito V, Amichetti M and Enrici RM: The role of fractionated radiotherapy and radiosurgery in the management of patients with craniopharyngioma. Neurosurg Rev 32: 125$132,2009$.

23 Murphy ES and Suh JH: Radiotherapy for vestibular schwannomas: A critical review. Int J Radiat Oncol Biol Phys 79: 985-997, 2011.

24 Li X, Li Y, Cao Y, Li P, Liang B, Sun J and Feng E: Safety and efficacy of fractionated stereotactic radiotherapy and stereotactic radiosurgery for treatment of pituitary adenomas: A systematic review and meta-analysis. J Neurol Sci 372: 110-116, 2017. 
25 Jaffray DA: Image-guided radiotherapy: From current concept to future perspectives. Nat Rev Clin Oncol 9: 688-699, 2012.

26 Nguyen NP, Nguyen ML, Vock J, Lemanski C, Kerr C, VinhHung V, Chi A, Khan R, Woods W, Altdorfer G, D'Andrea M, Karlsson U, Hamilton R and Ampil F: Potential applications and image-guided radiotherapy for brain metastases and glioblastoma to improve patient quality of life. Front Oncol 3: 284, 2013.

27 Badakhshi H, Kaul D, Wust P, Wiener E, Budach V and Graf R: Image-guided stereotactic radiosurgery for cranial lesions: large margins compensate for reduced image guidance frequency. Anticancer Res 33:4639-4643, 2013. Erratum in: Anticancer Res 33: 5707, 2013.

28 Kudo T, Ono K, Furukawa K, Fujimoto S, Akagi Y, Koyama T, Hirokawa Y: Comparison of setup accuracy between ExacTrac $\mathrm{X}$-ray 6 dimensions and cone-beam computed tomography for intracranial and pelvic image-guided radiotherapy. Nihon Hoshasen Gijutsu Gakkai Zasshi 68: 1644-1651, 2012.

29 Yuh GE, Loredo LN, Yonemoto LT, Bush DA, Shahnazi K, Preston W, Slater JM and Slater JD: Reducing toxicity from craniospinal irradiation: Using proton beams to treat medulloblastoma in young children. Cancer J 10: 386-390, 2004.

30 Yoon M, Shin DH, Kim J, Kim JW, Kim DW, Park SY, Lee SB, Kim JY, Park HJ, Park BK and Shin SH: Craniospinal irradiation techniques: A dosimetric comparison of proton beams with standard and advanced photon radiotherapy. Int J Radiat Oncol Biol Phys 81: 637-646, 2011.

31 Barney CL, Brown AP, Grosshans DR, McAleer MF, de Groot JF, Puduvalli V, Tucker SL, Crawford CN, Gilbert MR, Brown PD and Mahajan A: Technique, outcomes, and acute toxicities in adults treated with proton beam craniospinal irradiation. Neuro Oncol 16: 303-309, 2014.
32 Amichetti M, Cianchetti M, Amelio D, Enrici RM and Minniti G: Proton therapy in chordoma of the base of the skull: A systematic review. Neurosurg Rev 32: 403-416, 2009.

33 Amichetti M, Amelio D, Cianchetti M, Enrici RM and Minniti G: A systematic review of proton therapy in the treatment of chondrosarcoma of the skull base. Neurosurg Rev 33: 155-165, 2010.

34 Bishop AJ, Greenfield B, Mahajan A, Paulino AC, Okcu MF, Allen PK, Chintagumpala M, Kahalley LS, McAleer MF, McGovern SL, Whitehead WE and Grosshans DR: Proton beam therapy versus conformal photon radiation therapy for childhood craniopharyngioma: Multi-institutional analysis of outcomes, cyst dynamics, and toxicity. Int J Radiat Oncol Biol Phys 90: 354-361, 2014.

35 Ronson BB, Schulte RW, Han KP, Loredo LN, Slater JM and Slater JD: Fractionated proton beam irradiation of pituitary adenomas. Int J Radiat Oncol Biol Phys 64: 425-434, 2006.

36 Halasz LM, Bussière MR, Dennis ER, Niemierko A, Chapman $\mathrm{PH}$, Loeffler JS and Shih HA: Proton stereotactic radiosurgery for the treatment of benign meningiomas. Int $\mathrm{J}$ Radiat Oncol Biol Phys 81: 1428-1435, 2011.
Received September 10, 2018

Revised September 19, 2018

Accepted September 21, 2018 